\title{
$\beta$-catenin expression and claudin expression RTT $T$ pattern as prognostic factors of prostatic cancer progression
}

\author{
Attila M. Szász ${ }^{*+}$, Péter Nyirády ${ }^{\ddagger}$, Attila Majoros ${ }^{\ddagger}$, Attila Szendrõi ${ }^{*}$, \\ Miklós Szûcs ${ }^{*}$, Eszter Székely ${ }^{+}$, Anna-Mária Tõkés ${ }^{+}$, Imre Romics $^{\dagger}$ and \\ Janina Kulka ${ }^{+}$
}

*Department of Pathology, Brigham and Women's Hospital, Harvard Medical School, Boston, MA, USA, ${ }^{+2}$ nd

Department of Pathology and ${ }^{\ddagger}$ Department of Urology, Semmelweis University, Budapest, Hungary

Accepted for publication 28 May 2009

AMS and NP contributed equally to this work

\section{OBJECTIVE}

To investigate the patterns of expression of the junctional proteins $\beta$-catenin and claudins in different prognostic groups of patients with prostatic cancer, to determine their value as prognostic markers.

\section{PATIENTS AND METHODS}

We evaluated the samples of 30 patients who had a radical prostatectomy for organconfined cancer (pT2NOMO), men with clinically advanced cancer, and a control group with benign prostatic hyperplasia. Using immunohistochemistry applied to tissue microarrays, each group was evaluated for claudin- $1,-2,-3,-4,-5,-7,-8$ and -10 , and $\beta$-catenin expression.

\section{RESULTS}

There were differences among the three groups in the expression of claudin-1 $(P=0.001),-2(P=0.014),-3(P=0.027),-4$ $(P=0.001),-8(P=0.001)$ and $\beta$-catenin $(P=0.002)$, regardless of Gleason score. By contrast, claudin $-5,-7$ and -10 patterns were not significantly different among the groups. Furthermore, claudin-1 $(P=0.014)$ and $-4(P=0.004)$ could be used to distinguish between those patients who had metastases and those who did not.

\section{CONCLUSIONS}

The pattern of claudin expression could be a novel diagnostic marker in re-classifying adenocarcinomas, and an additional sensitive predictive factor for a clinically poor prognosis. Our results suggest that patients with organ-confined and advanced cancer are subsets with distinct claudin expression profiles, and that claudin-4 is related to cellular differentiation in prostate cancer, which is not only the receptor molecule for the Clostridium perfringens enterotoxin, and thus a theoretical future therapeutic target for prostate cancer, but also a marker of progression.

\section{KEYWORDS}

prostate, carcinoma, marker, progression, claudin, $\beta$-catenin

\section{INTRODUCTION}

Prostate cancer is one of the main causes of cancer-related mortality among men in Western societies, but knowledge of its aetiology and pathogenesis is still insufficient. Although, prostatic cancer often starts 'silently' and produces few early symptoms, after the widespread use of serum PSA testing it has been increasingly reported at earlier stages. In localized prostate cancer in men in good general health, with $>10$ years of life-expectancy, radical prostatectomy (RP) is the preferred treatment. Furthermore, RP might also provide a cure for well-selected patients with locally advanced prostate cancer [1]. However, it is still not possible to recognize who is at high risk of tumour recurrence after primary local therapy, and thus will not benefit from surgery [2]. Currently there are more promising indicators to distinguish between surgically curable and oncologically treatable prostate cancers, but there is still no optimum factor found which provides an accurate prognosis.

$\beta$-Catenin is a member of the Wnt signal pathway, which has been characterized and represents key targets for potential anticancer agents [3]. Wnt glycoproteins comprise a family of extracellular signalling ligands. Down-regulation of $E$-cadherin and $\beta$ catenin was found in the metastatic prostate cancer phenotype [4], which might be a significant factor in the genesis of bone metastases. However, the results did not appear to be reflected by expression in the primary tumours [5]. Experiments show that blocking certain components of the Wnt pathway is feasible and is an important way to inhibit the signalling required for cancer stem cells.

Claudins are 20-27-kDa proteins [6] that contribute to the formation of tight junctions [7], regulate paracellular transport [8] and signal transduction [9]. To date, 24 members of the claudin family have been described [10]. The expression of claudins show an organ-specific pattern [11], and is affected in several pathological processes, e.g. cell growth, carcinogenesis and tumour progression [12]. 
Claudin expression in the prostate was investigated by several groups; the expression of claudin-3 and -4 in the epithelium of prostate cancer [13], regulation of the expression of PSA by claudin-7 [14], and the expression pattern in mouse prostate [15]. Recent reports also indicate the importance of these proteins in prognosis, e.g. loss of claudin- 1 and -7 and expression of claudins3 and -4 have been shown to correlate with prognostic variables in prostatic adenocarcinomas $[16,17]$, and therapeutically, claudin-4 is the receptor molecule of Clostridium perfringens enterotoxin [18].

In the present study we investigated claudin expression patterns from a diagnostic and prognostic perspective, finding different expression of these molecules in prostatic adenocarcinomas which did not differ significantly in histological appearance on pathological evaluation, but have strikingly different behaviour on clinical follow-up.

\section{PATIENTS AND METHODS}

We analysed the claudin expression pattern in 30 Caucasian patients divided into three prognostic groups; 10 had clinically advanced (CA) or metastatic prostate cancer with a Gleason score of $\geq 7$ and a serum PSA level of $>100 \mathrm{ng} / \mathrm{mL}$. Seven of these patients had already had multiple bone metastases on their first urological examination. One of these men also developed liver and retroperitoneal metastases besides bone involvement. After histological confirmation of prostatic carcinoma, all were immediately administered total androgen blockade and bisphosphonates, followed by taxanes in case of hormone refractory status (treated according the valid European Association of Urology guideline). However, all of these patients died within 3 years of their first visit. The specimens in the CA group were taken as biopsies, at least six from each patient from both sides. The second group comprised 10 men who consecutively had RP for clinically and later pathologically confirmed organconfined (OC) prostate cancer (pT2NOMO), with a Gleason score of $\leq 7$ and $\geq 5$ years biochemical relapse- or progression-free survival. The third group comprised 10 patients with BPH (as a control), in complete urinary retention with large $(>60 \mathrm{~mL})$ prostates, who had a transvesical prostatectomy, and pathological evaluation showed BPH in the surgical specimen. The study was approved by the institutional

TABLE 1 The antibodies and conditions used for immunohistochemical reactions. The specific positive control tissues are shown next to the details of the antibodies

\begin{tabular}{llllll} 
Antibody & Manufacturer & ID\# & Dilution & $\begin{array}{l}\text { +ve control } \\
\text { tissue }\end{array}$ & $\begin{array}{l}\text { Threshold for morphometric } \\
\text { analysis (R/G/B) }\end{array}$ \\
\hline Claudin-1 & Zymed & $18-7362$ & $1: 80$ & Skin & $162 / 138 / 146$ \\
Claudin-2 & Zymed & $18-7363$ & $1: 80$ & Colon & $188 / 168 / 169$ \\
Claudin-3 & Zymed & $34-1700$ & $1: 80$ & Colon & $178 / 139 / 149$ \\
Claudin-4 & Zymed & $18-7341$ & $1: 100$ & Colon & $187 / 164 / 178$ \\
Claudin-5 & Zymed & $18-7364$ & $1: 120$ & Colon & $179 / 162 / 175$ \\
Claudin-7 & Zymed & $34-9100$ & $1: 100$ & Breast & $184 / 151 / 138$ \\
Claudin-8 & Zymed & $40-2600$ & $1: 80$ & Colon & $186 / 163 / 145$ \\
Claudin-10 & Zymed & $38-8400$ & $1: 60$ & Kidney & $182 / 157 / 161$ \\
$\beta$-catenin & BD Biosciences & 610154 & $1: 200$ & Bile duct & $119 / 66 / 42$
\end{tabular}

review board of Semmelweis University under licence \#185/2007.

The selected samples were examined histologically, and regions of interests were marked as agreed by two pathologists. The tissue microarrays (TMAs) were constructed of formalin-fixed and paraffin-embedded tissues, maintaining tumour cores $2 \mathrm{~mm}$ in diameter; two cores from each case were included. Sections ( $5 \mu \mathrm{m}$ thick) were obtained from the TMA blocks for immunohistochemical analysis. After deparaffinization steps, the slides were treated in a microwave oven in Target Retrieval Solution (S1699; Dako, Carpenteria, CA, USA) for 30 min for claudin retrieval, and Antigen Unmasking Solution (H3300; Vector Laboratories, Burlingame, CA, USA) was used for $23 \mathrm{~min}$ for $\beta$-catenin preparation. The immunohistochemical reactions were carried out in an automated immunostainer system (Model ES, Ventana Medical Systems Inc., Tucson, AZ, USA) with the solutions and steps according to the manufacturer's instructions. The claudin antibodies used are shown in Table 1. $\beta$-catenin immunostaining was performed according to the following protocol. The tissue samples were incubated with the $\beta$-catenin antibody (610154; Becton Dickinson Biosciences, San Jose, CA, USA) in Antibody Diluent solution (Vector Laboratories) for $24 \mathrm{~h}$, and the Envision+ system (K4001; Dako) was used for visualization of the reaction with 2,3diaminobenzidine chromogen (CMD401; CellMarque, Rocklin, CA, USA). The tissues were counterstained with Mayer's haematoxylin (00-8011; Zymed Laboratories Inc., South San Francisco, CA, USA). Positive controls (Table 1) and negative control tissues (with the omission of the primary antibodies) were included in every run. The results were evaluated semiquantitatively (scored from 0 to 3) before morphometric analysis.

The immunohistochemical reactions were digitized using a BX50F3 microscope, DP70 camera and software (v. 2.2.1.227; Olympus Optical Co., Tokyo, Japan); 10 representative images were taken of each case at $\times 200$ $(1320 \times 1024 \times 24$ bytes in pixels $)$ for later analysis. Although, the patients with CA only had biopsies available, and the $\mathrm{OC}$ and $\mathrm{BPH}$ groups had whole blocks for evaluation, TMA construction and the digitization of the same size of tissue surface area and morphometric analysis made it possible to compare these groups. The same tissue-handling protocol was applied to the biopsies and the tissue blocks at the pathological department. The morphometry was evaluated using OWin V3.0 software, which makes an objective quantification of the immunohistochemical results available (Leica Microsystems Imaging Solutions Ltd, Cambridge, UK). Binary transformation was used and threshold levels were selected on the positive control tissues (Table 1). Each digitized image was evaluated after correcting for possible errors occurring from heterogeneous tissue conformation, rejecting the lumen of vessels and ducts by manual selection. The units of expression were based on pixels.

For data analysis and statistical evaluation, all individual numerical values of the distinct measurements of the image analysis were included. Normality was tested, and Student's $t$-test and ANOVA used for the numerical morphometric values. Bivariate correlation analysis was used to correlate Gleason score and protein expression levels. A Cox proportional hazards regression model was 
used to evaluate tight-junction molecule expression with overall survival. The assumptions of the Cox model were tested and met. PSA serum levels, claudin and $\beta$ catenin expressions were evaluated as continuous variables. All statistical tests were two-sided, with $P<0.05$ considered to indicate statistical significance.

\section{RESULTS}

The pathological assessment and clinical follow-up data were available for all the patients; the mean age was 69.9 years in the
CA group, 60.4 years in the OC group and 66.6 years in the BPH group. The mean PSA level was 652.7 and $11.67 \mathrm{ng} / \mathrm{mL}$ in groups $C A$ and $\mathrm{OC}$, respectively. In the $\mathrm{BPH}$ group there was no evidence of prostatic carcinoma by a DRE and the presence of a catheter would have affected the PSA level. The patient characteristics are shown in Table 2.

The claudins showed membranous positivity, and unless otherwise noted, and only those reactions that showed the acceptable pattern were analysed by morphometry. Claudin-1 had significantly lower expression in group CA

TABLE 2 Basic patient characteristics, as the mean (SD)

\begin{tabular}{lllll} 
Group & N patients & Age, years & PSA level, $\mathrm{ng} / \mathrm{mL}$ & Mean Gleason score (sum) \\
\hline CA & 10 & $69.9(3.8)$ & $652.7(288.4)$ & $8.3(0.36)$ \\
OC & 10 & $60.4(2.2)$ & $11.67(1.4)$ & $5.0(0.42)$ \\
BPH & 10 & $66.6(0.9)$ & - & -
\end{tabular}

and $\mathrm{OC}$ than in the $\mathrm{BPH}$ group $(P=0.001)$. In the latter group the expression was striking in the basal cell layer. There was no significant difference between the $\mathrm{CA}$ and $\mathrm{OC}$ groups $(P=0.629)$. Claudin-2 also had a lower expression level in the $\mathrm{CA}$ and $\mathrm{OC}$ groups than in the BPH group $(P=0.014)$. Again, granular and mostly cytoplasmic staining was a peculiarity for this molecule, and it had a similar pattern to claudin-1 in the basal cell layer. The CA and OC groups did not differ in expression for claudin $(P=0.976)$.

Claudins-3 and -4 had higher expression in the CA group than in the $\mathrm{OC}$ and $\mathrm{BPH}$ groups $(P=0.027,0.009$; and $P=0.001$ and 0.002 , respectively). The pattern for claudin- 5 was similar to that for claudin-1 and -2 , and was noted in the endothelium of the vessels. The intensity of staining was lower in the CA and OC groups $(P=0.517)$. The CA group showed a more vascularized pattern than the $\mathrm{OC}$ and BPH groups (Fig. 1).

FIG. 1. Immunohistochemical images of the expression of the evaluated claudin-1,-2,-3 and -4 . The left column shows the CA group, the middle the OC group, and the right column the BPH group. Row 1, claudin-1; row 2, claudin-2; row 3, claudin-3; row 4, claudin-4 expression. The graphs on the right show the expression levels. $\times 200$.
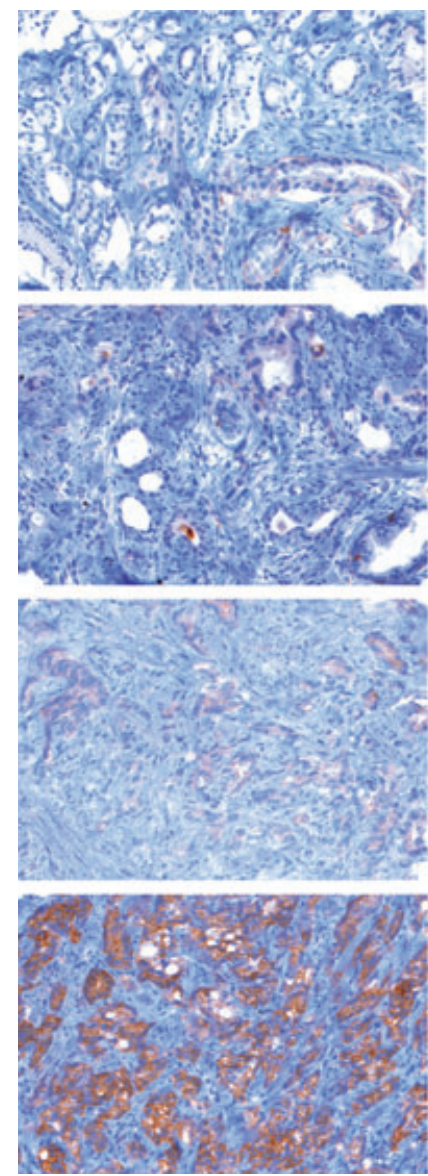
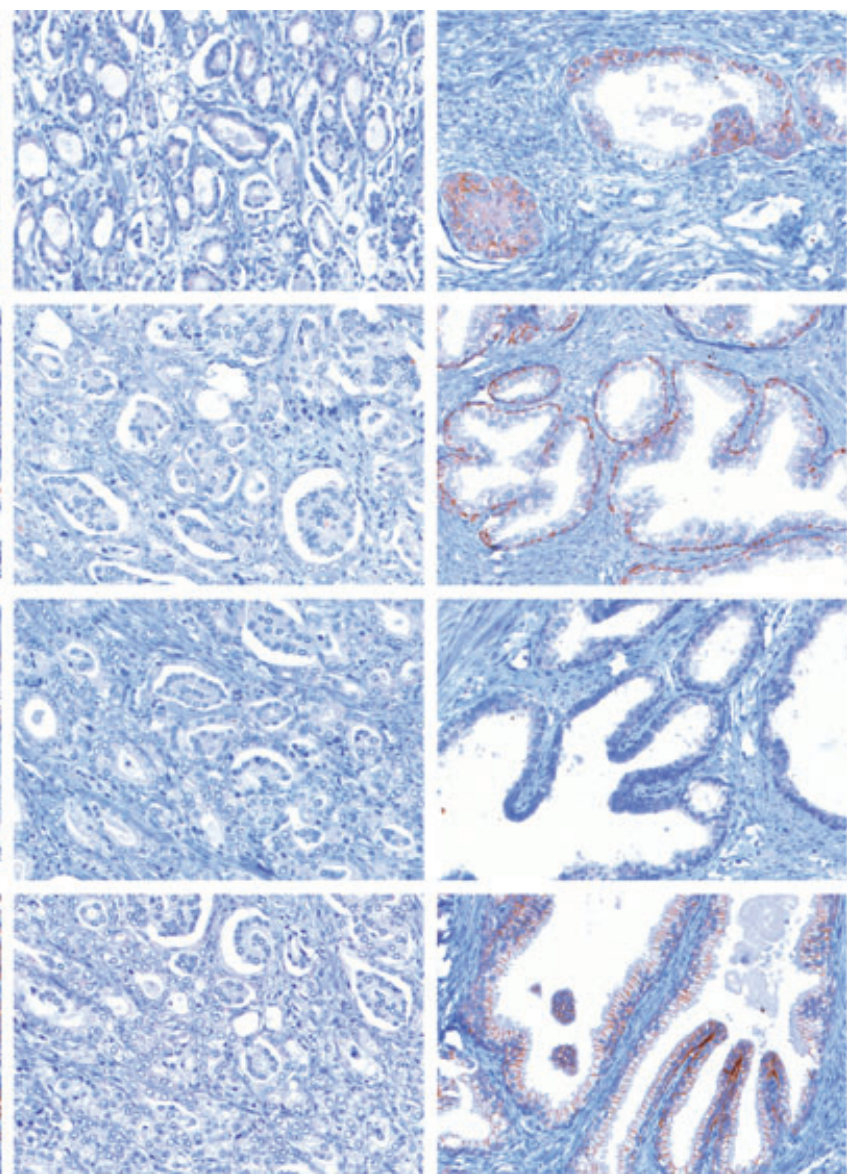

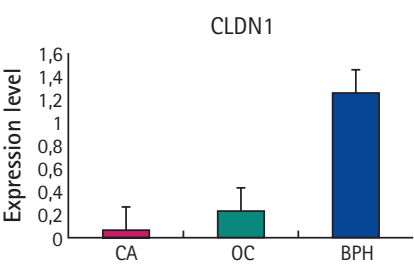

CLDN2

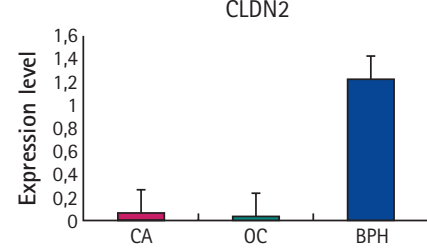

CLDN3

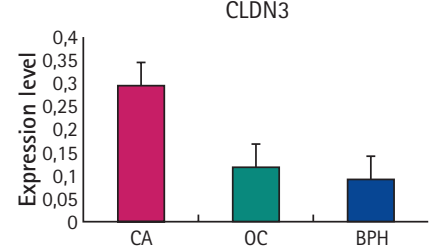

CLDN4

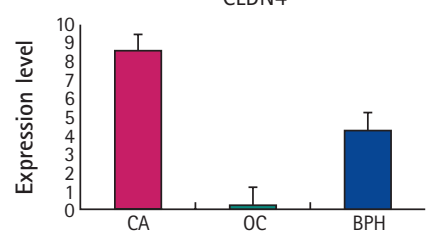


TABLE 3 The mean (SEM) of protein expression, morphometric values and scores in the three groups. Immunohistochemistry was scored as described in the Methods. The morphometric data represent pixel aspect ratios according to the percentage of positively stained cells. The CA, OC and BPH groups were compared using ANOVA and posthoc Tukey comparisons (morphometry) and Mann-Whitney tests (scoring).

\begin{tabular}{|c|c|c|c|c|c|c|c|c|c|c|c|}
\hline \multirow[b]{2}{*}{ Stain } & \multicolumn{5}{|c|}{ Morphometry } & \multicolumn{6}{|l|}{ Scoring } \\
\hline & Groups & Mean (SEM) & ANOVA & vs $\mathrm{BPH}^{*}$ & vs $O C^{*}$ & Mean (SEM) & ANOVA & vs $\mathrm{BPH}^{*}$ & vs $0 C^{*}$ & vs $\mathrm{BPH}+$ & vs $\mathrm{OCt}$ \\
\hline \multirow[t]{3}{*}{ Claudin-1 } & $\mathrm{CA}$ & $0.070(0.018)$ & - & $<0.001$ & 0.629 & $0.2(0.1)$ & & $<0.001$ & 0.001 & $<0.001$ & 0.003 \\
\hline & OC & $0.228(0.062)$ & $<0.001$ & $<0.001$ & & $1.2(0.2)$ & $<0.001$ & 0.099 & & 0.105 & \\
\hline & BPH & $1.258(0.167)$ & & & & $1.7(0.2)$ & & & & & \\
\hline \multirow[t]{3}{*}{ Claudin-2 } & CA & $0.060(0.007)$ & & $<0.001$ & 0.976 & 0.0 & & $<0.001$ & 0.486 & $<0.001$ & 0.481 \\
\hline & OC & $0.040(0.012)$ & $<0.014$ & $<0.001$ & & $0.2(0.1)$ & $<0.001$ & $<0.001$ & & $<0.001$ & \\
\hline & BPH & $1.220(0.098)$ & & & & $1.4(0.2)$ & & & & & \\
\hline \multirow[t]{3}{*}{ Claudin-3 } & CA & $0.293(0.075)$ & & 0.009 & 0.027 & $2.3(0.2)$ & & $<0.001$ & $<0.001$ & $<0.001$ & $<0.001$ \\
\hline & OC & $0.116(0.028)$ & 0.007 & 0.946 & & $0.2(0.1)$ & $<0.001$ & 0.075 & & 0.105 & \\
\hline & BPH & $0.097(0.009)$ & & & & $0.8(0.2)$ & & & & & \\
\hline \multirow[t]{3}{*}{ Claudin-4 } & CA & 8.454 (1.394) & & 0.002 & $<0.001$ & $2.5(0.2)$ & & 0.003 & $<0.001$ & 0.009 & $<0.001$ \\
\hline & $\mathrm{OC}$ & $0.186(0.040)$ & $<0.001$ & 0.003 & & $0.2(0.1)$ & $<0.001$ & 0.006 & & 0.009 & \\
\hline & BPH & $4.210(0.386)$ & & & & $1.3(0.3)$ & & & & & \\
\hline \multirow[t]{3}{*}{ Claudin-5 } & CA & $0.516(0.122)$ & & $<0.481$ & 0.394 & $0.2(0.1)$ & & $<0.327$ & 1.000 & $<0.421$ & 1.000 \\
\hline & $\mathrm{OC}$ & $2.255(0.505)$ & $<0.517$ & $<0.261$ & & $0.2(0.1)$ & $<0.451$ & $<0.285$ & & $<0.641$ & \\
\hline & $\mathrm{BPH}$ & $6.548(0.709)$ & & & & $1.9(0.2)$ & & & & & \\
\hline \multirow[t]{3}{*}{ Clauding-7 } & CA & $2.275(0.390)$ & & 0.561 & 0.811 & $1.5(0.2)$ & & 0.527 & 0.749 & 0.353 & 0.579 \\
\hline & $O C$ & $2.733(0.733)$ & 0.588 & 0.921 & & $1.7(0.2)$ & 0.547 & 0.930 & & 0.739 & \\
\hline & BPH & $3.008(0.364)$ & & & & $1.8(0.2)$ & & & & & \\
\hline \multirow[t]{3}{*}{ Claudin-8 } & CA & $0.017(0.008)$ & & 0.017 & 0.988 & $0.1(0.1)$ & & $<0.001$ & 1.000 & 0.001 & 1.000 \\
\hline & OC & $0.023(0.007)$ & 0.001 & 0.019 & & $0.1(0.1)$ & $<0.001$ & $<0.001$ & & 0.001 & \\
\hline & BPH & $0.203(0.109)$ & & & & $1.1(0.2)$ & & & & & \\
\hline \multirow[t]{3}{*}{ Claudin-10 } & CA & $0.088(0.023)$ & & 0.134 & 0.823 & 0.0 & & 0.412 & 0.800 & 0.143 & 0.739 \\
\hline & $\mathrm{OC}$ & $0.019(0.014)$ & 0.100 & 0.767 & & $0.1(0.1)$ & 0.403 & 0.153 & & 0.280 & \\
\hline & BPH & $0.005(0.001)$ & & & & $0.4(0.2)$ & & & & & \\
\hline \multirow[t]{3}{*}{$\beta$-catenin } & CA & $8.211(2.974)$ & & 0.078 & 0.616 & $1.3(0.3)$ & & 0.263 & 0.706 & 0.190 & 0.393 \\
\hline & OC & 5.055 (1.098) & 0.009 & 0.008 & & $1.0(0.3)$ & 0.067 & 0.059 & & 0.035 & \\
\hline & BPH & $15.430(2.288)$ & & & & $1.9(0.2)$ & & & & & \\
\hline
\end{tabular}

Claudin-7 staining was most prominent on the sides of the cells; the expression was localized to the cell membranes in all cases. There was no significant difference among the groups ( $P=0.588)$. However, claudin-8 staining was almost totally negative in both group of adenocarcinomas, while positive in $\mathrm{BPH}(P=0.001)$. The expression was mostly apparent at the level of tight junctions at the border of the apical and lateral membrane of the epithelial cells. Claudin-10 expression was not different in the examined groups.

$\beta$-catenin had a high level of expression in $\mathrm{BPH}$, and a lower level in the CA and OC groups $(P=0.002)$. There was heterogeneity of expression in the carcinomas, especially in the CA group; Table 3 shows the scoring and morphometric data.
Claudin-1 had a greater expression in carcinomas with a lower Gleason score; carcinomas that had a lower score but were CA did not express claudin-1 $(r=0.548$, $P=0.023)$. Claudin-2 was almost absent in malignant cells, and was not related to Gleason grade ( $r=-0.376, P=0.137)$. Claudin-3 and -4 were expressed highly in those carcinomas having higher Gleason scores and CA, while these claudins were almost absent in the $\mathrm{OC}$ group ( $r=0.572, P=0.016$; and $r=0.739$, $P=0.001$, respectively). Claudin-5 was only weakly expressed in clinically poorly behaving (CA) carcinomas $(r=-0.712, P=0.001)$, but was notable in the small vessels (Fig. 2). Claudin-7, -8 and -10 did not correlate with Gleason grade, and $\beta$-catenin had no correlation with Gleason grade or any other claudin protein expression.
The expression pattern of the selected molecules was then correlated with the presence of distant metastases. Seven of the patients in the CA group had already been diagnosed with bone metastases, and one of them also developed liver and retroperitoneal metastases. When comparing all the patients from all the three groups (22 and seven; one patient had no data, screening was therefore pending and he was excluded from this analysis), claudin-1 $(P=0.014)$ and -4 $(P=0.004)$ expression could be used to distinguish between those patients who had distant recurrence and those had not. When comparing all the invasive adenocarcinomas (12 and seven), only claudin-4 was expressed by the cells in a significantly different manner $(P=0.022)$; Fig. 3 shows the expression levels of these two proteins. 
FIG. 2. Immunohistochemical images of the expression of the evaluated claudin-5,-7,-8 and $\beta$-catenin. The left column shows the CA group, the middle the OC group and the right the BPH group. Row 1, claudin-5 (note the expression in the small vessels); row 2, claudin-7; row 3, claudin-8; row 4, $\beta$-catenin expression. The graphs on the right show the expression levels as columns. $\times 200$.
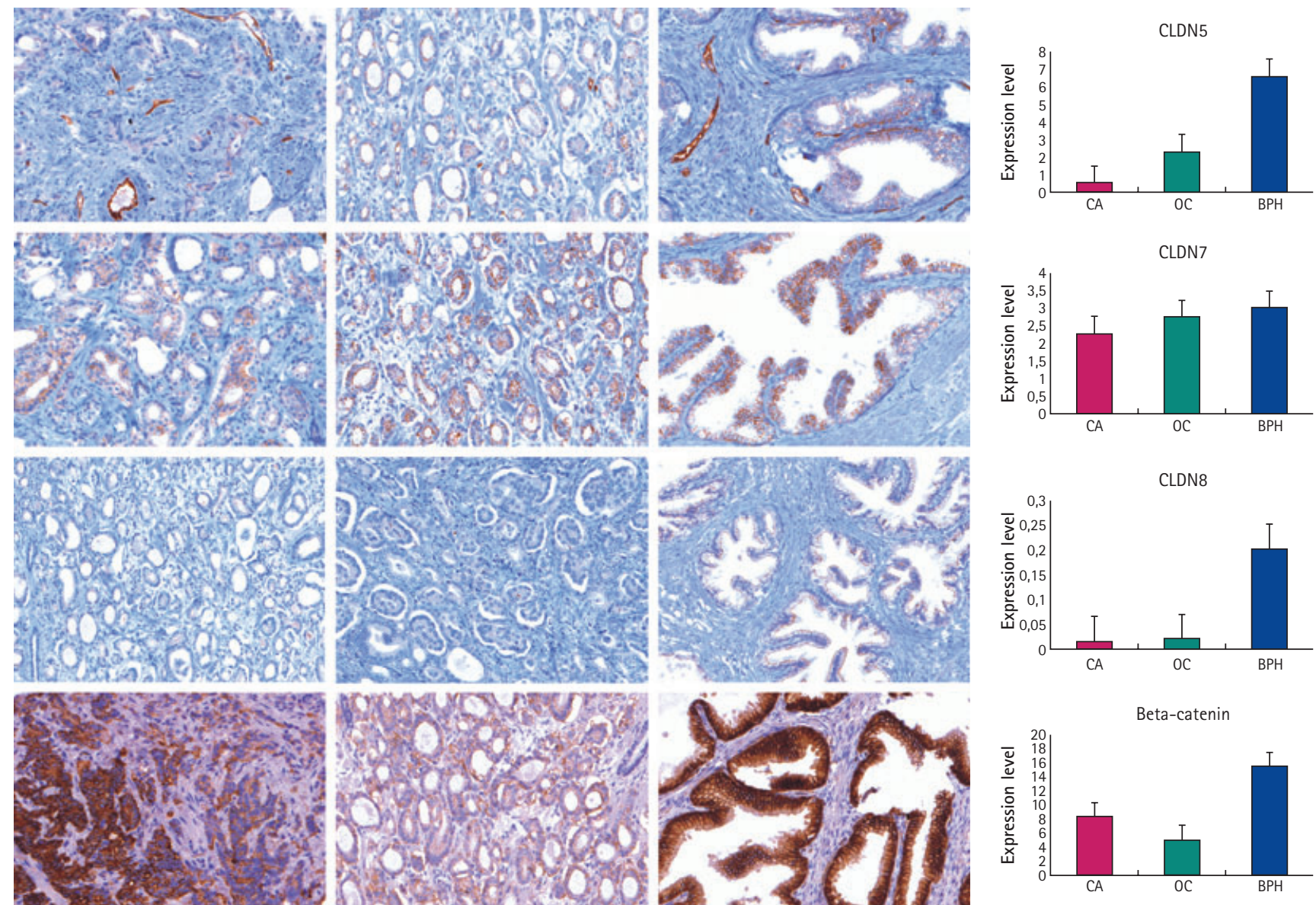

FIG. 3. A plot showing the expression in nonmetastatic prostatic disease (from the OC, $C A$ and BPH groups, 22 cases; red) and metastatic prostatic carcinomas (seven cases from group $C A$; green) for the mean surface area of claudin-1 $(\mathrm{P}=0.014)$ and claudin-4 $(\mathrm{P}=0.004)$ positive tumour cell membranes.

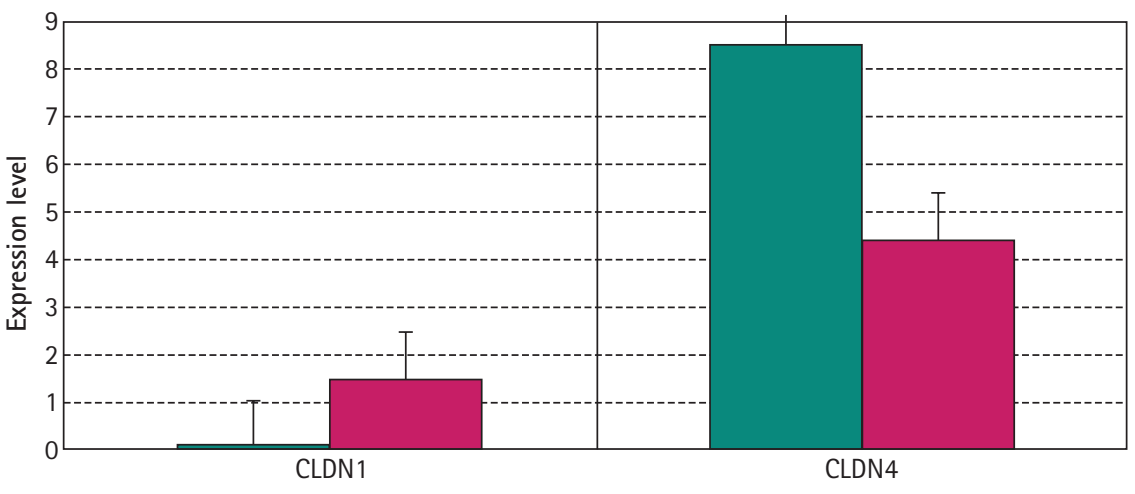

Tight junction protein

The expression of the tight-junction molecules was analysed together with the time to oncological failure. The primary endpoint of the study was overall survival.
Based on the reclassification, the expression of claudin-1 and -4 and overall survival were calculated to compare the examined groups. Both claudin-1 and -4 were able to predict survival of the groups with different outcomes (Fig. 4).

\section{DISCUSSION}

Claudins are important in the regulation of integrity and function of tight junctions, and might also influence tumorigenesis. The pattern of claudin expression of the prostate was reported previously, and is generating more interest $[16,17]$. However, the value of claudins as diagnostic markers or prognostic and/or predictive factors has not been investigated in depth. Therefore, the aim of the present study was to determine whether claudins could be useful not only in the pathological differential diagnosis of prostatic cancer, but also in the clinical setting in the prediction of prognosis.

We showed that claudin-1 was almost absent in the groups CA, while claudin-4 levels were higher in this group. In agreement with our 
findings, Sheehan et al. [16] concluded that the immunohistochemical expression and prognostic significance of claudins are variable in prostatic adenocarcinomas, with consistently lower expression of claudin-1. By contrast, the expression of claudin-3 and -4 correlated significantly with advanced-stage tumours and recurrence. In their study the immunohistochemical reactions were scored semiquantitatively and the expression of claudins in prostate cancer was compared to that of adjacent structures. By contrast, in the present study we evaluated claudin expression compared to $\mathrm{BPH}$ as a morphometric baseline value.

We found that the Gleason score correlated with the expression of claudin-1, -3 and -4 ; as did Vare et al. [19]. According to these results, it could be interpreted that claudin-4 is expressed by the less differentiated cells and this might theoretically be a novel therapeutic approach, as claudin-4 is able to bind the Clostridium perfringens enterotoxin [13]. Tõkés et al. [20], investigating claudin expression in breast cancer, found that the lower the grade of the tumour, the lower was the expression of claudin-4. Our results suggest that patients in group CA and $\mathrm{OC}$ are subsets with distinct claudin expression profiles, and that claudin-4 is related to cellular differentiation in prostate cancer, being re-expressed with progression. We found similar changes in the expression of claudin-4 in invasive carcinomas of the breast [21].

The expression pattern of claudin-1 was compared with that of the basal cell-specific markers p63 and high molecular weight cytokeratin in a study of normal prostatic epithelium, BPH, prostatic intraepithelial neoplasia and prostate adenocarcinoma [17]. There was pronounced claudin-1 expression in the basal cell layer, with no staining in luminal cells in benign prostatic epithelium. Claudin-1 immunohistochemistry was also suggested to be a new diagnostic tool for distinguishing malignant from benign lesions of the prostate. In the present study we found similar localization and expression of claudin-1 and -2; we also used BPH tissue as a control and investigated further claudin classes [16].

Claudin-7 expression was localized to the membranes of the epithelial cells in prostatic neo- and hyperplasia. This molecule might be of interest, as Wang et al. [22] showed that claudin-7 can up-regulate both the androgen receptor and PSA, and the membrane protein claudin-7 can act together with junctional adhesion molecule- $A$ in regulating a particular but unknown protein which is required for the expression of both androgen receptor and PSA. In the present study we could not correlate claudin-7 expression with serum PSA levels, possibly because too few patients were examined.

Gain-of-function and loss-of-function mutations have provided evidence that $\beta$ catenin is essential in development and tumorigenesis [23]. Not only $\beta$-catenin but also the activation of other components of the $\mathrm{Wnt} / \mathrm{\beta}$-catenin pathway in differentiated mammary epithelium and prostate epithelium of transgenic mice can induce neoplasia and transdifferentiation to squamous metaplasia [3]. We found that $\beta$-catenin expression was weaker in $\mathrm{OC}$ carcinomas, while its reexpression was detected in CA disease. There might be heterogeneity in the primary tumour, and the clones of different behaviour have different $\beta$-catenin expression. Previous studies did not provide unequivocal results, and describe both loss and the high expression of $\mathrm{E}$-cadherin and $\beta$-catenin in prostate carcinoma metastases $[4,5,24]$, but were mostly unable to reproduce the results in the primary carcinomas.

By investigating the claudin expression profile of tumours tending towards metastasis, we found that loss of claudin-1 and highly expressed claudin-4 was a significant combined profile in patients with metastatic prostate cancer. From the perspective of cancer biology, it is interesting that breast and prostate cancers with a poor prognosis overexpress claudin-4. Claudin-4 was recently reported to be a marker highly overexpressed in both primary and metastatic prostate cancer [25]. By contrast, and similarly to Sheehan et al. [16] and Vare et al. [19], our group found claudin-4 to be expressed in those carcinomas that had a higher Gleason grade and the capacity to metastasize. Because the Gleason scores of the CA group and the OC group overlap, we cannot separate them based on this sole feature.

We found higher claudin-5 expression in the endothelial cells of the capillary system. Nevertheless, our study was unable to confirm neo-angiogenetic gain of claudin- 5 expression, which might be due to the few
FIG. 4. The Cox proportional hazards model of survival after randomization stratified by claudin-4 expression. The patients with prostatic carcinoma and high claudin-4 expression (green) had a significantly shorter survival than those with low expression (red) and the BPH group (blue-dotted). The time of survival is in months. (Red and blue dotted lines are superimposed.)

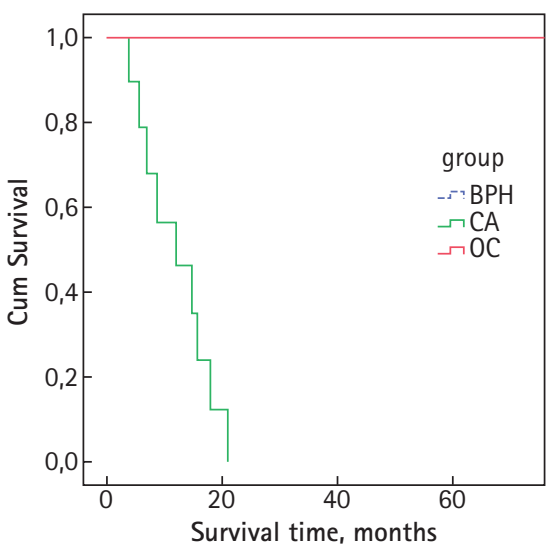

samples assessed. Changes in claudin-4 protein expression might be a more important feature of different kinds of adenocarcinomas from different organs than previously thought [26]. Our findings also suggest that future target-orientated therapies could possibly be used in the treatment of metastatic prostate cancer.

In conclusion, as in other recent studies, we showed that claudin-1 expression could be a novel prognostic marker to distinguish benign and malignant prostatic lesions. In parallel with our previous findings, claudin-4 seems to be important in cellular differentiation, and might be used as a marker of the progression of prostatic adenocarcinomas in a clinical setting. However, additional studies with more patients are required before our findings could be used in practice.

\section{ACKNOWLEDGEMENTS}

The authors thank Azumah Francisné for the preparation of slides and immunohistochemistry. AMSz was partly supported by the Babics Antal Foundation and the generosity of the Rosztoczy Foundation. Grant support: ETT-049/2006.

\section{CONFLICT OF INTEREST}

None declared. 


\section{REFERENCES}

1 Van Poppel H, Goethuys H, Callewaert P, Vanuytsel L, Van de Voorde W, Baert L. Radical prostatectomy can provide a cure for well-selected clinical stage T3 prostate cancer. Eur Uro/ 2000; 38: 372-9

2 Khan MA, Partin AW. Management of high-risk populations with locally advanced prostate cancer. Oncologist 2003; 8: 259-69

3 Morin PJ. Beta-catenin signaling and cancer. Bioessays 1999; 21: 1021-30

4 Junior JP, Srougi M, Borra PM, Dall' Oglio MF, Ribeiro-Filho LA, Leite KR. E-cadherin and beta-catenin loss of expression related to bone metastasis in prostate cancer. Appl Immunohistochem Mol Morphol 2008 (Epub ahead of print).

5 Bryden AA, Hoyland JA, Freemont AJ, Clarke NW, Schembri Wismayer D, George NJ. E-cadherin and beta-catenin are down-regulated in prostatic bone metastases. BJU Int 2002; 89: 400-3

6 Morita K, Furuse M, Fujimoto K, Tsukita S. Claudin multigene family encoding four-transmembrane domain protein components of tight junction strands. Proc Natl Acad Sci USA 1999; 96 : 511-6

7 Furuse M, Fujita K, Hiiragi T, Fujimoto K, Tsukita S. Claudin-1 and -2: novel integral membrane proteins localizing at tight junctions with no sequence similarity to occludin. J Cell Biol 1998; 141: 1539-50

8 Heiskala M, Peterson PA, Yang Y. The roles of claudin superfamily proteins in paracellular transport. Traffic 2001; 2: 93-8

9 Yamamoto T, Kojima T, Murata M et al. IL-1 beta regulates expression of Cx32, occludin, and claudin-2 of rat hepatocytes via distinct signal transduction pathways. Exp Cell Res 2004; 299: 427-41

10 Katoh M, Katoh M. CLDN23 gene, frequently down-regulated in intestinal- type gastric cancer, is a novel member of CLAUDIN gene family. Int J Mol Med 2003; 11: 683-9

11 Hewitt KJ, Agarwal R, Morin PJ. The claudin gene family: expression in normal and neoplastic tissues. BMC Cancer 2006; 6: 186

12 Tzelepi VN, Tsamandas AC, Vlotinou HD, Vagianos CE, Scopa CD. Tight junctions in thyroid carcinogenesis: diverse expression of claudin-1, claudin4, claudin-7 and occludin in thyroid neoplasms. Mod Pathol 2008; 21: 22-30

13 Long $\mathrm{H}$, Crean $\mathrm{CD}$, Lee $\mathrm{WH}$, Cummings OW, Gabig TG. Expression of Clostridium perfringens enterotoxin receptors claudin-3 and claudin-4 in prostate cancer epithelium. Cancer Res 2001; 61 : 7878-81

14 Zheng JY, Yu D, Foroohar M etal. Regulation of the expression of the prostate-specific antigen by claudin-7. J Membr Biol 2003; 194: 187-97

15 Sakai N, Chiba H, Fujita H etal. Expression patterns of claudin family of tight-junction proteins in the mouse prostate. Histochem Cell Biol 2007; 127 : 457-62

16 Sheehan GM, Kallakury BV, Sheehan CE, Fisher HA, Kaufman RP Jr, Ross JS. Loss of claudins- 1 and -7 and expression of claudins- 3 and -4 correlate with prognostic variables in prostatic adenocarcinomas. Hum Pathol 2007; 38: 564-9

17 Krajewska M, Olson AH, Mercola D, Reed JC, Krajewski S. Claudin-1 immunohistochemistry for distinguishing malignant from benign epithelial lesions of prostate. The Prostate 2007; 67: 90710

18 Gardiner RA, Landers KA, Burger MJ, Scells $B$, Samaratunga M, Lavin MF. Claudin 4: a new therapeutic target for prostate cancer? BJU Int 2005; 95: 1

19 Vare P, Loikkanen I, Hirvikoski P, Vaarala MH, Soini Y. Low claudin expression is associated with high
Gleason grade in prostate adenocarcinoma. Oncol Rep 2008; 19: 25-31

20 Tokes AM, Kulka J, Paku S et al. Claudin$1,-3$ and -4 proteins and mRNA expression in benign and malignant breast lesions: a research study. Breast Cancer Res 2005; 7: R296-305

21 Kulka J, Szasz A, Nemeth Z et al. Expression of tight junction protein claudin-4 in basal-like breast carcinomas. Pathol Oncol Res 2009; 15: 59-64

22 Wang 0, Zheng JY, Kreth J et al. Regulation of prostate-specific antigen expression by the junctional adhesion molecule A. Urology 2009; 73: 111925

23 Miyoshi K, Hennighausen L. Betacatenin: a transforming actor on many stages. Breast Cancer Res 2003; 5: 638

24 Saha B, Arase A, Imam SS et al. Overexpression of $\mathrm{E}$-cadherin and betacatenin proteins in metastatic prostate cancer cells in bone. Prostate 2008; 68: 78-84

25 Landers KA, Samaratunga H, Teng L et al. Identification of claudin-4 as a marker highly overexpressed in both primary and metastatic prostate cancer. Br J Cancer 2008; 99: 491-501

26 Facchetti F, Lonardi S, Gentili F et al. Claudin 4 identifies a wide spectrum of epithelial neoplasms and represents a very useful marker for carcinoma versus mesothelioma diagnosis in pleural and peritoneal biopsies and effusions. Virchows Arch 2007; 451: 669-80

Correspondence: Janina Kulka, 2nd

Department of Pathology, Semmelweis University, H-1091 Budapest, Ulloi ut 93, Hungary.

e-mail: kj@korb2.sote.hu

Abbreviations: CA, clinically advanced; OC, organ-confined; $\mathrm{RP}$, radical prostatectomy; TMA, tissue microarray. 